\title{
Actuarial Technique of Modelling the Out of Pocket with Deductible and Stop Loss in Health Care Insurance
}

\author{
Ogungbenle Gbenga Michael \\ Department of Actuarial Science, Faculty of Management Sciences, \\ University of Jos, Nigeria.
}

Email:moyosiolorun@gmail.com

\begin{abstract}
The paper is intended to numerically estimate health insurance out of pocket spending from the perspective of the enrollees in order to inform whether health insurance scheme pricing policy delivers value to the enrollees. We study the implications of financing health care insurance using a classification model for health care insurance under the framework of deductibles and stop loss. The paper is designed for health underwriting professionals, particularly those who consult for health insurance schemes. This is essential given the form and emergence of treating enrollees fairly, based on regulations that impact on actuarial consultants advising on health insurance scheme product lines. The objectives of this paper are to demonstrate actuarial methods for estimating (i) total health expenses (ii) out of pocket costs (iii) reimbursements by third party insurance. (iv) examine the actuarial implications of the chosen model. In computing the out of pocket and reimbursement, the Pitacco's model was adopted because of its relatively computational superiority and the fact that both deductible and stop loss form its core parameters. Our results show that there is a positive relationship between total health expense and the share funded by out of pocket spending. As the health expenditure increases, the share funded by out of pocket progressively increases.
\end{abstract}

Keywords: Deductible, Enrollee, Out of pocket, Premium, Stop loss

\section{Introduction}

Health insurance scheme is one of the varied strategies used in funding health care program so as to hedge against health uncertainties. A core function of health insurance program is to provide insurance protection against the out-of-pocket (OOP) health expenditures. A large amount of health service in Nigeria is paid out as OOP by enrollees who have been trapped by abject poverty because of paying health care costs. The current techniques of obtaining indirect computation of out of pocket from data reported in demographic survey of household expenditure by employing national insurance scheme estimates of household consumption data or by integrating data from various sources such as survey of economic activities and household survey are not actuarially reliable, inadequate and not mathematically justified. Oftentimes, the survey methods may depend on proxy respondents who elicit information on behalf of other enrollees and who have not been personally interviewed. By using actuarial procedures, this paper investigates OOP estimation procedures and exposure of enrollees with health expenditures following the implementation of Pitacco's health transformation model. Each time the bill of a hospital treatment varies over observed enrollees, it could be socially significant to treat only few enrollees and not everyone. Where an enrollee is not fully certain of his health condition ex-ante, he could be interested in buying full health insurance covering treatments for high-benefit patients while he abandons treatment for low benefit patients. However, where potential medical disorders are noticed but not ascertained, health insurance underwriters could have problems denying health care to low benefit patients. Deductibles therefore would push low benefits patients to show their health condition due to the imposition of marginal health cost on treatment but at a price of incomplete risk-sharing and consequently lump-sum indemnities could induce low benefit patients to abandon treatment. 
Ogungbenle Gbenga Michael/ Mathematical Techniques of Modelling the Out of Pocket with Deductible ...

Financial risks in health insurance expenses have aggravating intensity consequences on health conditions because, the irrational phobia of not paying up health insurance bills could compel an employee postpone health care at the risk of aggravating the health status. In Bhattacharjya \& Sapra (2008) and Faden, VialleValentin, Ross-Degnan \& Wagner (2011), health insurance scheme is a technique of advance funding of health care expenditure through contributions payable into a common fund such as national health insurance scheme (NHIS) to reimburse whole or part of health insurance services specified in an insurance policy. Following Pakhare (2009), it is essentially to get rid of uncertain risk of severity of the enrollee by combining a number of enrollees who are similarly exposed and listed in a common pool. The basic parameters in health insurance are advance payment of contributions, pooling of funds and qualification for benefits based on contributions or career not dependent on any income test that is, observing how the computed results behave when some underlying assumptions change. In Pakhare (2009), we infer that the basic ingredients in national health insurance scheme are advance payment which allows enrollees to pay when healthy and which eliminates the difficulties at the time of critical health conditions and risk pooling based on the probability that only few enrollees will become ill. In view of Rao (2004), Pakhare (2009), it applies to all kinds of insurances indemnifying for losses caused by body injuries or illness or for cost of medical treatment as a result of sickness or body injury. Intensive health insurance care is a necessary policy instrument used to mitigate outof-pocket spending by poor enrollees because the lower out-of-pocket spending among the vulnerable poor enrollees is quite functional related to the almost exclusive use of health services rendered by health care providers.

Health insurance could apply to a restricted or wider spectrum of health services and could make provision for part or full reimbursement of the costs on specified services. Benefits usually depends on being qualified for specified health services or indemnity of the enrollees for specified medical costs. Insurance of hospital costs is a common type of private health insurance cover although most medical coverage provide protection against critical medical costs but avoid the financial and administrative risk associated with insuring small costs. Health insurance reimbursements assist in enhancing the efficiency of health insurance supply systems. This could help to use the opportunities in negotiating leverage given by the dense populations and the capacity to add or remove specified indemnity from the reimbursement menu to negotiate advantageous cost for the enrollees. Many advance economies such as Italy, Japan and the USA have established varied degrees of restrictive models applying evidence based and cost-efficient benchmarks to enhance quality service delivery and reduce costs. A reimbursement menu consisting of grey area of health care conditions could provide broad benefit which is convenient to manage but where a reimbursement menu is restricted, enrollees could ultimately buy medicines which are not included in formularies at high out-of-pocket costs. Therefore, a high level of quality services would constitute a generic strategy to attain cost-efficient health insurance use. Defined constituents of all-inclusive health program to improve generics use, may comprise enrollees, health provider education and information, financial incentives to attain target rates of generics, tiered enrollees' copayments and differential co-payment levels as cost indications to enrollees. Differential co-payments could be employed to establish financial incentives for enrollees to demand generics or more cost-efficient treatment. The majority of the evidence in literature borders on the influence of reimbursement design. There is scary numerically based evidence on cost selection or contribution management techniques. We found no evidence in Nigeria evaluating mathematical out-of-pocket health insurance dynamics with deductible as core variable often employed by underwriters in developing economies such as actuarial based contribution rate.

\section{A Survey of Casualty Preliminaries}

One of the methods of cost-sharing mechanism in health care program which has taken its root recently in developing economies is paying deductibles in which case the insured enrollees carefully utilize health care services while avoiding many unnecessary potential costs. The most relevant advantage of deductibles is the reduction in utilization of varying health services, low health benefit claims culture by the insured enrollees and increase in financial profit margin of health insurance organization and make health insurance program solvent. However, a core disadvantage of deductible relates to increase in out-of-pocket burdens. The philosophy behind deductibles is that it is believed that if health insurers continues to pay for these bills from the commencement of health care costs, this would increase uncontrollable utilization of medical services and 
hence increases health care costs. Through the application of the numerous cost containment vehicles, health products and pricing are differentiated as the risk cost varies. A compulsory excess on enrollees' claims is specified to eliminate the number of small nuisance medical expense claims that would possibly result in significant fraction of the total number of claims and a high proportion of administrative cost. In an ordinary deductible, the enrollee consents to cover a defined sum of a claim before the insurance firm reimburses. We observe in Ramjee, et al. (2014), Kaplan (2015) and Kaplan \& Ranchod (2015) that the defined sum paid out of pocket $\delta$ is the deductible so that the insurance firm can assume coverage for the remainder loss $(Y-\delta)$. Following Faden, et al. (2011), health insurance deductibles are applicable to every covered loss in relation to the defined agreement. The rationale is to discourage excessive claims, eliminate handling and processing costs thereby reducing moral hazards. Enrollees tend to assume larger risks which could increase the probability of loss associated with insured peril because the enrollee knows the insurance firm will definitely incur cost.

Following Schlesinger (1985), Jack \& Ormiston (1999), Tse (2009), Thogersen (2016), Woodard \& Yi (2018) and Ogungbenle, et al. (2020), the two types of payments payable by the insurance firm are (i) the payment per loss and (ii) payment per payment. The payment per loss random variable represents the losses over which payment is effected and losses on which payment is less than the agreed deductible. We suppose that $\mathrm{Y}$ denotes the loss incurred to the enrollee and $\mathrm{X}$ denotes the claim payable by the insurance firm.

$$
X_{L}=(Y-\delta)^{+}=\left\{\begin{array}{c}
0, \text { if } \quad Y<\delta \\
(Y-\delta), \text { if } \quad Y>\delta
\end{array}\right.
$$

$X_{L}$ is the shifted variable since values less than $\delta$ are not done away with and all losses are shifted by $\delta$. The payment per payment $X_{P}$ becomes unbounded at the time the insurer does not legally incur any claim but only consists of losses over which claims have been paid. $X_{P}$ becomes the excess loss variable because claims less than $\delta$ will not be reported and losses exceeding $\delta$ are shifted by $\delta$

$$
X_{P}=\left\{\begin{array}{c}
\text { undefined, if } \quad Y \leq \delta \\
(Y-\delta), \text { if } \quad Y>\delta
\end{array}\right.
$$

Following Tse (2009), Ogungbenle, et al. (2020) and Thogerson (2016) though $Y$ is required to be continuous, the probability distribution of $X_{L}$ is partially continuous and partially discrete with the discrete distribution centered at $X=0$ for $X<\delta$ but the continuous part is concentrated over the interval $X>0$ where $X>\delta$

$$
P\left(X_{L}=0\right)=P(Y \leq \delta)=F_{Y}(\delta)
$$

Thus, the probability that losses are less than or equal to the deductible coincides with the probability that there is no payment.

Let us define

$$
X_{L}=(Y-\delta)
$$

for the continuous aspect of the probability distribution, then the probability distribution of $X_{L}$ is defined as

$$
f_{X_{L}}(x)=\left\{\begin{array}{c}
F_{Y}(\delta), \text { if } \quad x=0 \\
f_{Y}(x+\delta), \text { if } \quad x>0
\end{array}\right.
$$

$X_{P}=X_{L} / Y>\delta$, the payment per payment random variable represents the payment per loss random variable conditional on the loss greater than the deductible. The density of $X_{P}$

$$
f_{X_{P}}(x)=\frac{f_{X}(x+\delta)}{1-F_{X}(\delta)} ; x>0 .
$$


The probability distribution of payment per payment random variable and payment per loss variable are given as follows

$$
\begin{aligned}
& F_{X_{L}}(x)=\left\{\begin{array}{c}
F_{Y}(\delta), \text { if } \quad x=0 \\
F_{Y}(x+\delta), \text { if } \quad x>0
\end{array}\right. \\
& F_{X_{P}}(x)=\frac{F_{X}(x+\delta)-F_{X}(\delta)}{1-F_{X}(\delta)} \text { for } x>0 \\
& S_{X_{P}}(x)=\frac{S_{X}(x+\delta)}{S_{X}(\delta)} \text { for } x>0 \\
& E\left(X_{L}\right)^{m}=\int_{\delta}^{\infty}(x-\delta)^{m} f_{X}(x) d x \\
& E\left(X_{P}\right)^{m}=\frac{\int_{\delta}^{\infty}(x-\delta)^{m} f_{X}(x) d x}{1-F_{X}(\delta)}=\frac{E\left(X_{L}\right)^{m}}{S_{X}(\delta)}
\end{aligned}
$$

Professional underwriters serving as health consultants to employers assume technical role as those of health insurance actuaries in advising employers on issues associated with pricing and employers' risk. In Asheim, et al. (2004), individual enrollees differ in the degree of inborn ability and likelihood of falling sick. Given information on the probability of sickness and an enrollee's inborn ability is very weak, competitive health insurance result in screening in the form of deductible. In health insurance, uncertainties may not be limited to financial quantities since actuarial risk is a function of risk and probability. In Duncan (2018) we observe the following definition,

$$
\text { risk }=\mathfrak{R}=h(\alpha, \theta)
$$

where $\alpha$ is the probability of a loss occurring and $\theta$ is the magnitude of loss. Based on the above definition of risk function, the risk cost premium from our perception could be obtained as follows.

$\mathfrak{R}=\alpha \times E \times A \times(1+\zeta)(1+\rho)$

$\Re$ is the risk cost and could be obtained from health expenditure as $\frac{\text { health care expenditure }}{\text { Population of enrollees }}$

$E$ is the projected expected expense per day of being hospitalised

$A=\frac{\text { Total expenses }}{\text { Number of bed days }}$ is the average stay in hospital duration as in-patient.

$\rho$ is the rate of inflation which can be modeled by retail price index to obtain inpatient's estimated expenses up to a defined period of time.

$\alpha$ is the probability of a claim occurring.

$\zeta$ is the loading factor characterizing skewness $\frac{(X-\mu)^{3}}{\sigma^{3}}$ of severity of each age-group

Health liability cash flows possess measurable implicit uncertainties because of volatility of claims experience, challenges in estimating the future reasonable projections associated with scheme variations, the lack of appropriate experience studies, variations between the enrollees \& regulatory environment and differences in interest crediting practices as well as variations in contributions as a result of changing number of enrollees.

We are very much keen in the varying levels of financial loss occurring as a result of varying levels and the imposed cost on the enrollees. The risk defined above requires that losses be quantitatively measured and that the probability of loss occurring be obtained through numerical procedures. In Pitacco (2017a), Pitacco 
(2017b), the measurement of health risk requires recognizing approaches in which the magnitude of losses and the associated probability of occurrence could be reduced. A common health care risk which health insurance managers face borders on risks associated with-- pricing. By accepting to absorb the pricing risk, health insurance contract indemnifies numerous health related contracts in exchange for a defined monthly contribution. In view of Barber, Lorenzoni and Ong (2019), it should be observed that the experience of health insurers is associated with the number of claims and size of claims.

Though, individual claims could be of moderate magnitude, the insurer may experience a highly disproportionate volume so that the risk consisting of loss and the probability of loss occurring could be defined in terms of claims or and severity. Since health care insurance contracts generally do not signify the amount payable on a defined service, it is therefore necessary that the magnitude of loss be approximately obtained. We infer from Barber, Lorenzoni and Ong (2019), that health insurance contracts agree to indemnify those health services ordered by the medical doctors which are considered medically necessary by the insurance firm for the insured's condition but cases of exclusion clauses in health insurance are not all that common. Numerous employer sponsored health schemes are characterized by administrative services only where the employer absorbs the financial risk and the third-party administrators such as insurance firm assumes the administrative risk at a defined price while the employer still retains the financial risk in form of severity. The individual patients experience health conditions in distinct levels hence a series of factors leading to unpredictable severities on a given set of health conditions advise medical doctors to treat the conditions differently. However, many health systems associated with health providers and payers often lead to similar methodologies and medical treatments being indemnified at varying amounts. In setting the health care cost for the enrollees, the underwriter assumes definite probability densities associated with frequencies of the claims. We infer in Manning \& Marquis (1996), Martin (2001) that the health insurer expects that the pool of risk will improve on the average the approximated risk level. While a few enrollees will be more expensive, some will cost less than the mean projected level. A correct actuarial examination of the insurance pool necessitates that underwriting standard be established at such a point that the actuarial distribution of insured's risk in the pool numerically estimates the distribution of the expected pricing of the policy holders. It is observed that high or low risk profiles could lead to claims exceeding the expected pricing. Bench marks to access facilities may consist of terms that the enrollees show evidence of sound health as at the time of registration or be subject to exclusion of claims clauses on pre-existing basis.

Furthermore, they could be subjected to a restriction on high cost procedures. The underwriting procedures should be capable of recognizing enrollees under critical health risk conditions. Though medical underwriting is applicable, the underwriting procedure may be asymmetrical in that the enrollees are certainly more aware of their health conditions than the underwriter. The underwriting risks comprise costs of uncertain risks while the pricing risk is associated with certain risk. However, underwriting risk may occur when incepting a fresh enrollee and usually influenced by the rate and conditions at which the entrants are incepted. The frequencies of claims are often affected by varying endogenous and exogenous parameters including but not limited to enrollees, their conditions and their strict compliance with their medical advice and often the training which health providers have undergone coupled with benchmark-based health standard practices. Furthermore, the emergence of new medical technology or new services and challenging access to the current available health services constitutes a significant factor. There are varying factors causing some kind of behaviour from both enrollees and health care service providers. The providers' behaviour is associated with using pay for service and value-based contract while the enrollees' behavior is associated with using defined interventionist programs though deductibles and co-payment are basically applied. In order to examine how health insurance impacts on enrollees, it is necessary to observe the complex nature of actuarial models used in health insurance underwriting. The basic variables are premium costs, deductibles, copays and coinsurance. The co-insurance reduces unnecessary use of health services but is an incentive for the enrollees to look for low cost treatment. 
Inability to consult qualified health workers on critical health related issues or preventive \& curative care results in the emergence of health conditions which without health insurance scheme could consequently result in financial ruin. Enrollees may tend to opt out of health insurance due to its high cost, despite its role in maintaining good medical condition.

The monthly contribution paid to an insurer to buy a health policy is health insurance premium representing the basic source of earning for health insurance providers. In order to generate profit, health insurance providers charge commensurate premium and invest same than they indemnify for benefits. The premiums should be paid irrespective of whether an enrollee incurs medical expenses or not.

Generally speaking, employers release a large amount of their employees' contributions, however many enrollees contribute to premium costs. The employer's part of enrollees' health care premiums are exempted from national and state income taxes and the employee's part is often deducted before withholding and exempted from taxable income. Before any insurance coverage is accessed, the enrollees should pay deductible which represents an amount of medical costs, however the amount of deductible varies with scheme but it is usually defined as a yearly maximum sum and consequently the deductible is renewed yearly and so each year scheme holders pay fresh deductible charge although they could have paid the full deductible value in the past years. Usually, health insurance scheme with large deductible is designed with a smaller monthly premium while a policy with a smaller deductible comes with high premium. When an enrollee visits a doctor or perhaps needs an emergent treatment or in some cases buys prescription drug, he pays a fixed amount known as copay. Copay is subject to drastic increase for those with health maintenance organizationbased policies if they obtain medical treatment from medical practices outside their provider's network domain.

However, as health costs increases, health insurers tend to look for other techniques to cut down medical expenses. Consequently, they connive with doctors, hospitals and other health insurance providers which offer smaller rates giving room for insurers to shift the burden of savings to their enrollees. In order to share costs with enrollees, health care insurers apply copays and coinsurance policies.

Following Faden, et al. (2011), the copay regime could save fund but compound the complexity of health insurance. Enrollees typically should examine two sets of fees, the fees established for providers within their approved networks and those set beyond the networks. Generally, fees are much reduced when the insured consult approved providers. In Onwujekwe, et al. (2012), copays are usually chargeable each time you consult a medical provider though it varies and are imposed on tests, prescribed drugs, physical therapy and other covered expenses. Apparently, copay costs can increase sharply for enrollees with critical medical conditions under severe health conditions which are costly to treat. In order to address the amounts payable by enrollees, many policies have annual and lifetime maximum depending on scheme on out-of-pocket payments.

In practice as it is done in advanced economies an employee who has been enrolled in a deductible health insurance scheme should open a health insurance savings account. The Funds contributed to health savings account are not subjected to national income taxes provided it is used for legal health expenses such as co-insurance, co-pays and deductibles. Following Faden, et al. (2011), a health insurance deductible describes the sum of money payable out-of-pocket on healthcare services covered under health insurance scheme before plan commences to pay benefits for qualified expenses. The amount payable on health insurance deductible is dependent on the type of health insurance plan and covered benefits. When appraising health insurance cover, we need to knowledge of what deductible covers and items it does not cover since approved health care cost payable out-of-pocket is driven towards enrollee's plan's deductible for that year. However, co-payments earlier discussed are usually not subject to this rule. Co-payments should not be confused with coinsurance which is the sum payable on medical services, provided deductible conditions are satisfied and the scheme starts reimbursements. Co-insurance are proportions of the medical charges payable even after meeting the deductible conditions and the insurer is responsible for the remaining portion of the bill. The sum paid on deductible, co-payments and coinsurance are considered in the annual out-of-pocket maximum which is the maximum sum individual pays before the insurance scheme starts paying the full amounts. Where an enrollee 
may not meet a plan's deductible for the year, it is reasonable to consider smaller rates for care if he decides to self-pay instead of using insurance coverage. It is possible that health insurance providers may be willing to offer services at subsidized rate if one prefers to pay out-of-pocket but the risk would be severe during complications leading to health emergencies. The amount of the out-of-pocket expense limits tends to change from one insurance scheme to another. However, the same insurer may have changing levels of plan. Health plans may have an annual out-of-pocket upper limit and once the amount is satisfied, it will not be necessary to pay coinsurance or copays again for the covered medical expenses that one incurs.

\section{$3 \quad$ Basic Derivations In Stop Loss Insurance Function}

In stop loss insurance the ceding insurance firm assumes aggregate claim $Y$ under $\delta>0$

$$
C_{\delta}= \begin{cases}Y, \text { if } & Y \leq \delta \\ \delta, \text { if } & Y \geq \delta\end{cases}
$$

This is the retention of cedent. The reinsuring company reimburses the cedent(primary insurer) the aggregate claim value in excess of $\delta>0$

$$
R_{\delta}=\left\{\begin{array}{c}
0, \text { if } \quad Y \leq \delta \\
(Y-\delta), \text { if } \quad Y \geq \delta
\end{array}\right.
$$

The aggregate claim is then split as follows

$$
\sum=C_{\delta}+R_{\delta}
$$

$E(\Sigma)=E\left(C_{\delta}\right)+E\left(R_{\delta}\right)$

$E(\Sigma)=\int_{\delta}^{\infty} y f_{Y}(y) d y-\delta \operatorname{Pr}(y \geq \delta)+E\left(R_{\delta}\right)$

$E\left(R_{\delta}\right)=\int_{\delta}^{\infty} y f_{Y}(y) d y-\delta \operatorname{Pr}(y \geq \delta)$

and $E\left(C_{\delta}\right)=E(\Sigma)-\int_{\delta}^{\infty} y f_{Y}(y) d y-\delta \operatorname{Pr}(y \geq \delta)=E(\Sigma)-E\left(R_{\delta}\right)$

If the expense as a function of single risk for the cedent is $C_{\delta}$ and the premium $k_{\delta}$ is paid to the reinsurance firm, then, the income generated by the primary insurer is

$$
\Pi_{\delta}=E\left(C_{\delta}\right)+k_{\delta}=E(\Sigma)-E\left(R_{\delta}\right)+k_{\delta} \text { for } \delta>0
$$

Suppose the benefit reimbursed by the contract is $\mathrm{b}$ when the actual loss is $\ell$. The claim parameter having fixed deductible $\delta$ is $Z=I$ (claim) $b$ where,

$$
b=\left\{\begin{array}{c}
0, \text { if } \quad \ell<\delta \\
(\ell-\delta), \text { if } \quad \ell>\delta
\end{array}\right.
$$

The deductible raises up the likelihood of zero benefit but reduces the level of the continuous density function. Loss model is usually constructed based on present value functions having stochastic components. By the application of general risk criteria principle (RC) which bases decisions on expectation of loss, we have

$$
E(b)=(1-p) \int_{\delta}^{\infty} \ell f_{\ell}(\ell) d \ell-\delta \operatorname{Pr}(\ell>\delta)
$$

is the net single value where $f_{\ell}(\ell)$ is the density of the actual loss and $p+q=1$ 
Loss function for $\delta=I($ claim $) b-(1-p) \int_{\delta}^{\infty} \ell f_{\ell}(\ell) d \ell-\delta \operatorname{Pr}(\ell>\delta)$

Assuming $\Sigma \sim N\left(\mu_{\Sigma}, \sigma_{\Sigma}\right)$, then for any fixed $\delta>0$

$$
\begin{aligned}
& E\left(R_{\delta}\right)=\sigma_{\Sigma}\left[f\left(\frac{\delta-\mu_{\Sigma}}{\sigma_{\Sigma}}\right)\right]+\mu_{\Sigma}-\mu_{\Sigma} \phi\left(\frac{\delta-\mu_{\Sigma}}{\sigma_{\Sigma}}\right)+\delta \phi\left(\frac{\delta-\mu_{\Sigma}}{\sigma_{\Sigma}}\right)-\delta \\
& E\left(R_{\delta}\right)=\int_{\delta}^{\infty} y f_{Y}(y) d y-\delta \operatorname{Pr}(y>\delta)=\sigma_{\Sigma}\left[f\left(\frac{\delta-\mu_{\Sigma}}{\sigma_{\Sigma}}\right)\right]+\left(\mu_{\Sigma}-\delta\right)\left[1-\phi\left(\frac{\delta-\mu_{\Sigma}}{\sigma_{\Sigma}}\right)\right]
\end{aligned}
$$

$f($.$) is the probability density function and \phi($.$) is the cumulative distribution function$

The following theorem shows the relationship between the functional values of deductible \& coverage and the survival function at both $\delta$ and $\beta$. Moreover, it defines the extent to which the health insurance scheme is theoretically fair as the rate of deductible declines while increasing coverage. It imposes the condition that deductibles cannot continue to increase perpetually under any fair health insurance scheme.

\subsection{Theorem 1}

If $\delta$ is the deductible and $\beta$ is the health coverage. Suppose $g(\delta)$ is a monotonically decreasing function $g^{\prime}(\delta)<0$ and $h(\beta)$ is monotonically increasing function $h^{\prime}(\beta)>0$, then

$$
g(\delta) h(\beta)+\delta S_{Y}(\delta)-\beta S_{Y}(\beta)-\int_{\delta}^{\beta} f_{Y}(y) d y=0
$$

\section{Proof}

$$
\begin{aligned}
& g(\delta) h(\beta)=\int_{\delta}^{\beta}\left(1-F_{Y}(y)\right) d y \\
& g(\delta) h(\beta)=\int_{\delta}^{\beta}\left(1-F_{Y}(y)\right) d y=\beta-\delta-\int_{\delta}^{\beta} F_{Y}(y) d y
\end{aligned}
$$

where $S_{Y}(y)=\int_{y}^{\infty} f_{R}(r) d r$

Integrating by parts, we have

$$
\begin{aligned}
& \int_{\delta}^{\beta} y f_{Y}(y) d y=\left[y F_{Y}(y)\right]_{\delta}^{\beta}-\int_{\delta}^{\beta} F_{Y}(y) d y \\
& \Rightarrow \int_{\delta}^{\beta} F_{Y}(y) d y=\left[y F_{Y}(y)\right]_{\delta}^{\beta}-\int_{\delta}^{\beta} y f_{Y}(y) d y \\
& g(\delta) h(\beta)=\int_{\delta}^{\beta}\left(1-F_{Y}(y)\right) d y=\beta-\delta+\int_{\delta}^{\beta} y f_{Y}(y) d y-\left[y F_{Y}(y)\right]_{\delta}^{\beta} \\
& g(\delta) h(\beta)=\int_{\delta}^{\beta}\left(1-F_{Y}(y)\right) d y=\beta-\delta+\int_{\delta}^{\beta} y f_{Y}(y) d y-\left[\beta F_{Y}(\beta)-\delta F_{Y}(\delta)\right] \\
& g(\delta) h(\beta)=\int_{\delta}^{\beta}\left(1-F_{Y}(y)\right) d y=\beta-\delta+\int_{\delta}^{\beta} y f_{Y}(y) d y-\beta F_{Y}(\beta)+\delta F_{Y}(\delta)
\end{aligned}
$$


$g(\delta) h(\beta)=\delta F_{Y}(\delta)-\delta+\beta-\beta F_{Y}(\beta)+\int_{\delta}^{\beta} f_{Y}(y) d y$

$g(\delta) h(\beta)=\delta\left(F_{Y}(\delta)-1\right)+\beta\left(1-F_{Y}(\beta)\right)+\int_{\delta}^{\beta} f_{Y}(y) d y$

$g(\delta) h(\beta)=\beta S_{Y}(\beta)-\delta S_{Y}(\delta)+\int_{\delta}^{\beta} f_{Y}(y) d y$

By the hypothesis of the theorem

$$
\begin{aligned}
& g(\delta) h(\beta)+\delta S_{Y}(\delta)-\beta S_{Y}(\beta)-\int_{\delta}^{\beta} f_{Y}(y) d y=0 \\
& g^{\prime}(\delta) h(\beta)+S_{Y}(\delta)+\delta S_{Y}^{\prime}(\delta)+f_{Y}(\delta)=0 \\
& g^{\prime}(\delta) h(\beta)+S_{Y}(\delta)-\delta f_{Y}(\delta)+f_{Y}(\delta)=0 \\
& g^{\prime}(\delta) h(\beta)=f_{Y}(\delta)(\delta-1)-S_{Y}(\delta) \\
& g^{\prime}(\delta)=\frac{f_{Y}(\delta)(\delta-1)-S_{Y}(\delta)}{h(\beta)}<0
\end{aligned}
$$

Again,

$$
\begin{aligned}
& g(\delta) h^{\prime}(\beta)-S_{Y}(\beta)-\beta S_{Y}^{\prime}(\beta)-f_{Y}(\beta)=0 \\
& g(\delta) h^{\prime}(\beta)-S_{Y}(\beta)+\beta f_{Y}(\beta)-f_{Y}(\beta)=0 \\
& g(\delta) h^{\prime}(\beta)=S_{Y}(\beta)-\beta f_{Y}(\beta)+f_{Y}(\beta) \\
& g(\delta) h^{\prime}(\beta)=S_{Y}(\beta)-(\beta-1) f_{Y}(\beta) \\
& h^{\prime}(\beta)=\frac{S_{Y}(\beta)-(\beta-1) f_{Y}(\beta)}{g(\delta)}>0
\end{aligned}
$$

\section{Theorem 2}

If $Y \sim \operatorname{Lognormal}\left(\mu, \sigma^{2}\right)$, then

$$
\begin{aligned}
\delta g(\delta) h(\beta)= & {\left[E\left(Y_{\beta}\right)\left(Y_{\delta}\right)-\left(\exp \left(2 \mu+\frac{4 \sigma^{2}}{2}\right) \Phi\left(\frac{\log _{e} \delta-\mu-2 \sigma^{2}}{\sigma}\right)\right)-\delta \beta\left(S_{Y}(\beta)\right)\right] } \\
& -\delta\left[\exp \left(\mu+\frac{\sigma^{2}}{2}\right) \Phi\left(\frac{\log _{e} \beta-\mu-\sigma^{2}}{\sigma}\right)-\exp \left(\mu+\frac{\sigma^{2}}{2}\right) \Phi\left(\frac{\log _{e} \delta-\mu-\sigma^{2}}{\sigma}\right)\right] \\
& +\left[\beta-\delta-(\beta-\delta) \exp \left(\mu+\frac{\sigma^{2}}{2}\right) \Phi\left(\frac{\log _{e} \beta-\mu-\sigma^{2}}{\sigma}\right)\right]
\end{aligned}
$$




\section{Proof}

Equivalently, $g(\delta) h(\beta)$ can be defined as follows

$$
\begin{aligned}
& g(\delta) h(\beta)=\int_{\delta}^{\beta}(y-\delta) f_{Y}(y) d y+\int_{\beta}^{\infty}(\beta-\delta) f_{Y}(y) d y \\
& g(\delta) h(\beta)=\int_{\delta}^{\beta} y f_{Y}(y) d y-\int_{\delta}^{\beta} \delta f_{Y}(y) d y+\int_{\beta}^{\infty}(\beta-\delta) f_{Y}(y) d y
\end{aligned}
$$

In general insurance business, the amount payable for a scheme with a policy limit structure is defined as $Y_{\Gamma}$ where the policy holder will be indemnified up to pre-defined value $\Gamma$. Defining $\min \{Y, \beta\}=(Y \Lambda \beta)=Y_{\beta}$

$E[(Y \Lambda \beta)(Y \Lambda \delta)]=\int_{0}^{\delta} y^{2} f_{Y}(y) d y+\delta \int_{\delta}^{\beta} y f_{Y}(y) d y+\delta \beta\left(S_{Y}(\beta)\right)$

$\int_{\delta}^{\beta} y f_{Y}(y) d y=\frac{1}{\delta}\left[E[(Y \Lambda \beta)(Y \Lambda \delta)]-\int_{0}^{\delta} y^{2} f_{Y}(y) d y-\delta \beta\left(S_{Y}(\beta)\right)\right]$

$g(\delta) h(\beta)=\frac{1}{\delta}\left[E[(Y \Lambda \beta)(Y \Lambda \delta)]-\int_{0}^{\delta} y^{2} f_{Y}(y) d y-\delta \beta\left(S_{Y}(\beta)\right)\right]-\int_{\delta}^{\beta} \delta f_{Y}(y) d y+\int_{\beta}^{\infty}(\beta-\delta) f_{Y}(y) d y$

where $\min \{Y, \beta\}=(Y \Lambda \beta)=Y_{\beta}$

$g(\delta) h(\beta)=\frac{1}{\delta}\left[E\left[\left(Y_{\beta}\right)\left(Y_{\delta}\right)\right]-\int_{0}^{\delta} y^{2} f_{Y}(y) d y-\delta \beta\left(S_{Y}(\beta)\right)\right]-\int_{\delta}^{\beta} \delta f_{Y}(y) d y+\int_{\beta}^{\infty}(\beta-\delta) f_{Y}(y) d y$

By the assumption in (ii) $y$ has a lognormal distribution structure and parameters $\mu, \sigma$ and density function $f_{Y}(y)$, then

$$
\int_{0}^{\delta} y^{2} f_{Y}(y) d y=\exp \left(2 \mu+\frac{4 \sigma^{2}}{2}\right) \Phi\left(\frac{\log _{e} \delta-\mu-2 \sigma^{2}}{\sigma}\right)
$$

and $\int_{0}^{\delta} y f_{Y}(y) d y=\exp \left(\mu+\frac{\sigma^{2}}{2}\right) \Phi\left(\frac{\log _{e} \delta-\mu-\sigma^{2}}{\sigma}\right)$

for any real $\delta>0$ hence substituting the densities appropriately (44) and (45) into (43) we have,

$$
\begin{aligned}
g(\delta) h(\beta)= & \frac{1}{\delta}\left[E\left[\left(Y_{\beta}\right)\left(Y_{\delta}\right)\right]-\left[\exp \left(2 \mu+\frac{4 \sigma^{2}}{2}\right) \Phi\left(\frac{\log _{e} \delta-\mu-2 \sigma^{2}}{\sigma}\right)\right]-\delta \beta\left(S_{Y}(\beta)\right)\right] \\
& -\delta\left[\int_{0}^{\beta} f_{Y}(y) d y-\int_{0}^{\delta} f_{Y}(y) d y\right]+(\beta-\delta)\left[\int_{0}^{\infty} f_{Y}(y) d y-\int_{0}^{\beta} f_{Y}(y) d y\right] \\
g(\delta) h(\beta)= & \frac{1}{\delta}\left[E\left[\left(Y_{\beta}\right)\left(Y_{\delta}\right)\right]-\exp \left(2 \mu+\frac{4 \sigma^{2}}{2}\right) \Phi\left(\frac{\log _{e} \delta-\mu-2 \sigma^{2}}{\sigma}\right)-\delta \beta\left(S_{Y}(\beta)\right)\right] \\
- & \delta\left[\exp \left(\mu+\frac{\sigma^{2}}{2}\right) \Phi\left(\frac{\log _{e} \beta-\mu-\sigma^{2}}{\sigma}\right)-\exp \left(\mu+\frac{\sigma^{2}}{2}\right) \Phi\left(\frac{\log _{e} \delta-\mu-\sigma^{2}}{\sigma}\right)\right] \\
& +\left[\beta-\delta-(\beta-\delta) \exp \left(\mu+\frac{\sigma^{2}}{2}\right) \Phi\left(\frac{\log _{e} \beta-\mu-\sigma^{2}}{\sigma}\right)\right]
\end{aligned}
$$


The out-of-pocket remains the biggest source of health insurance finance in underdeveloped economies such as Nigeria but nevertheless the most difficult in computation. The difficulty level in computing the out of pocket numerically can potentially set back the acceptability of health insurance scheme with the resultant effect that health regulators may not approve of the appropriateness of the resulting policy goal. Therefore, it is necessary to apply a new actuarial methodology in computing the out-of-pocket (OOP). The total expenditure incurred by individual consists of OOP and health insurance cost while OOP is often the dominating factor. The model on out of pocket and re-imbursement developed by Pitacco (2017a) in (48), (50) and (51) have been found more acceptable because of its computational superiority and the fact that health deductible and stop loss variables constitute its core parameters. Pitacco's models seem more actuarially reliable than the indirect computational figures derived from data often reported in demographic health surveys of household expenditure by reference to national health insurance scheme computations of household consumption which do not adopt deductible and stop loss actuarially. In practice, health insurance is usually underwritten under general insurance business though it is a life insurance product hence it bears the features of both life and general insurance where deductibles mostly apply and this justifies the adoption of the model. Furthermore, as at the time of writing, there does not seem to be found in actuarial literature any other similar model like Pitacco's which considers both deductible and stop loss. However, a gap found in the model is that it does not address the issues relating to expected out-of-pocket and expected reimbursements which form part of our contribution to literature.

We define the following notations.

$E$ is the expense

$P$, the proportional deductible is the fraction of the eligible medical expenses which the enrollees pay after satisfying the flat deductible.

$\delta$, the flat deductible is a predetermined sum which the enrollee pays out-of-pocket before the insurer provides for part cover on the remaining eligible expenses.

$\Sigma$ is the stop loss. Stop loss value is the maximum the enrollee will pay out of pocket and could be referred either to every single claim or to the policy period. Usually, the underwriter retains aggregate claims under $\delta>0$ $\pi$, the out-of-pocket describes a method of health financing and represents amount paid to health service providers at the moment of service delivery. They usually assume a formal cost sharing mechanism or a type of direct payments and reference is often made to direct payments as the enrollee pays for complete value of health services not insured through any type of coverage. Whereas the formal cost sharing represents expenses on health services defined in the benefit but which are not covered in full.

$z$ is the benefit reimbursed by the underwriting company.

$$
\pi=\left\{\begin{array}{c}
E, \text { if } \quad E<\delta \\
P(E-\delta)+\delta, \text { if } \quad E \in[\delta, h) ; P \in(0,1] \\
\Sigma, \text { if } \quad E \geq h
\end{array}\right.
$$

Expenses $=($ Average Visit $) \times($ Average cost of every visit $)$

In the health insurance sector, the out-of-pocket costs are the portion of the fees that the insurance firm will not cover and which the enrollees must pay for on their own. They represent cost on medical care which would not be reimbursed by insurance comprising coinsurance, deductibles, co-insurance and co-payments for covered services together with other costs for non-covered services. Health insurance plans are subject to out-of-pocket maximum which represent caps on the amount of fees which an enrollee or a scheme holder can spend each year on covered healthcare expenses. The out-of-pocket expenses $\pi$ further represents the costs which individual enrollees pay from their own cash reserves and it is usually applied in describing an enrollee's work related expenses which 
could subsequently be reimbursed by the employer under a specific defined employer-approved procedure. Common instances of working-related out-of-pocket expenses are airfare, taxis, lodging and meals. From the insurance point of view, the out of pockets expense describes an enrollee's proportion of health insurance costs that comprises among other things the incurred sum on coinsurance, copays and deductibles. Health care insurance scheme has out-of-pocket caps which is the upper limit sum payable every year for covered healthcare expenses. Many health insurance schemes are issued to establish long time ceiling on how much the policy should reimburse an enrollee for medical costs, though it is not expected that there should be any ceiling on the amount an insurer pays to cover enrollee's medical expenses provided copays, coinsurance costs and deductibles conditions are satisfied and consequently insurers should be restrained from cancelling enrollee's policy for medical reasons.

$$
\begin{aligned}
z= & \left\{\begin{array}{c}
0, \text { if } \quad E<\delta \\
(1-P)(E-\delta), \text { if } \quad E \in[\delta, h) ; P \in(0,1] \\
E-\Sigma, \text { if } \quad E \geq h
\end{array}\right. \\
& {[\Sigma-(\delta-\delta P)]=P h }
\end{aligned}
$$

\subsection{The Actuarial Health Implications of the Model}

The followings are the consequences of the models (48), (50) and (51)

(i) The expected out of pocket is defined as follows

$$
E(\pi)=\int_{0}^{\delta} E f_{E}(E) d \pi+P \int_{\delta}^{h} E f_{E}(E) d E+(\delta-P \delta) \operatorname{Pr}(\delta<E<h)+\sum \times \operatorname{Pr}(E \geq \delta)
$$

(ii) The second moment of expected out of pocket is given as

$$
\begin{aligned}
E\left(\pi^{2}\right)= & \int_{0}^{\delta} E^{2} f_{E}(E) d \pi+P^{2} \int_{\delta}^{h} E^{2} f_{E}(E) d E+(\delta-P \delta)^{2} \operatorname{Pr}(\delta<E<h)+\sum^{2} \times \operatorname{Pr}(E \geq \delta) \\
\operatorname{Var}(\pi) & =E\left(\pi^{2}\right)-[E(\pi)]^{2} \\
\operatorname{Var}(\pi) & =\left[\int_{0}^{\delta} E^{2} f_{E}(E) d \pi+P^{2} \int_{\delta}^{h} E^{2} f_{E}(E) d E+(\delta-P \delta)^{2} \operatorname{Pr}(\delta<E<h)+\sum^{2} \operatorname{Pr}(E \geq \delta)\right] \\
& -\left[\int_{0}^{\delta} E f_{E}(E) d \pi+P \int_{\delta}^{h} E f_{E}(E) d E+(\delta-P \delta) \operatorname{Pr}(\delta<E<h)+\sum \operatorname{Pr}(E \geq \delta)\right]^{2}
\end{aligned}
$$

(iii) The expected reimbursement is given as follows

$$
E(z)=(1-P) \int_{\delta}^{h} E f_{E}(E) d E-(\delta-P \delta) \operatorname{Pr}(\delta<E<h)+\int_{h}^{\infty} E f_{E}(E) d E-\sum \operatorname{Pr}(E \geq h)
$$

(iv) The second moment of expected reimbursement is computed as

$$
\begin{aligned}
E\left(z^{2}\right)= & (1-P)^{2} \int_{\delta}^{h} E^{2} f_{E}(E) d E-(\delta-P \delta)^{2} \operatorname{Pr}(\delta<E<h)+\int_{h}^{\infty} E^{2} f_{E}(E) d E-\sum^{2} \operatorname{Pr}(E \geq h) \\
\operatorname{Var}(z) & =E\left(z^{2}\right)-[E(z)]^{2} \\
\operatorname{Var}(z) & =\left[(1-P)^{2} \int_{\delta}^{h} E^{2} f_{E}(E) d E-(\delta-P \delta)^{2} \operatorname{Pr}(\delta<E<h)+\int_{h}^{\infty} E^{2} f_{E}(E) d E-\sum^{2} \operatorname{Pr}(E \geq h)\right] \\
& -\left[(1-P) \int_{\delta}^{h} E f_{E}(E) d E-(\delta-P \delta) \operatorname{Pr}(\delta<E<h)+\int_{h}^{\infty} E f_{E}(E) d E-\sum \operatorname{Pr}(E \geq h)\right]^{2}
\end{aligned}
$$


Theorem 3: If $f(E)$ defines the density of $E$, then, $E(\pi)+E(z)=E(E)$

\section{Proof}

We recall immediately in equations (52) and (56) that

$$
\begin{aligned}
E(\pi)+E(z)= & {\left[\int_{0}^{\delta} E f_{E}(E) d \pi+P \int_{\delta}^{h} E f_{E}(E) d E+(\delta-P \delta) \operatorname{Pr}(\delta<E<h)+\sum \times \operatorname{Pr}(E \geq \delta)\right] } \\
& +\left[(1-P) \int_{\delta}^{h} E f_{E}(E) d E-(\delta-P \delta) \operatorname{Pr}(\delta<E<h)+\int_{h}^{\infty} E f_{E}(E) d E-\sum \operatorname{Pr}(E \geq h)\right] \\
E(\pi)+E(z)= & \int_{0}^{\delta} E f_{E}(E) d \pi+P \int_{\delta}^{h} E f_{E}(E) d E+\delta \operatorname{Pr}(\delta<E<h)-P \delta \operatorname{Pr}(\delta<E<h) \\
& +\sum \times \operatorname{Pr}(E \geq \delta)+\int_{\delta}^{h} E f_{E}(E) d E-P \int_{\delta}^{h} E f_{E}(E) d E-\delta \operatorname{Pr}(\delta<E<h) \\
& +P \delta \operatorname{Pr}(\delta<E<h)+\int_{h}^{\infty} E f_{E}(E) d E-\sum \operatorname{Pr}(E \geq h) \\
E(\pi)+E(z)= & \int_{0}^{\delta} E f_{E}(E) d \pi+\int_{\delta}^{h} E f_{E}(E) d E+\int_{h}^{\infty} E f_{E}(E) d E
\end{aligned}
$$

Observe that

$$
\begin{aligned}
& E(E)=\int_{0}^{\delta} E f_{E}(E) d \pi+\int_{\delta}^{h} E f_{E}(E) d E+\int_{h}^{\infty} E f_{E}(E) d E \\
& E(\pi)+E(z)=E(E) \\
& E(z)=E(E)-E(\pi)
\end{aligned}
$$

\section{Data Presentation and Analysis}

The data was collected from a primary health care provider referred by a health maintenance organization (HMO) under its administration in Jos South, Nigeria. We were allowed to extract relevant information on enrollees from the register of medical records such as number of visits and the charge per visit from JanuaryDecember, 2020 only on normal regular services covered by health insurance scheme. The expenditure was

\begin{tabular}{|c|c|c|c|c|}
\hline Medical & & & & \\
\hline Expenses & Out of pocket & $\begin{array}{l}\text { Re-imbursement } \\
\text { Benefit }\end{array}$ & $\begin{array}{l}\text { Out of pocket }+ \\
\text { Benefit }\end{array}$ & Percentage \\
\hline 500.00 & 500.00 & 0.00 & 500.00 & 1.00 \\
\hline
\end{tabular}
then estimated monthly per enrollee depending on the average number of times the enrollee has visited the health care provider for each month and the average cost of every visit using equation (49), then finally the total annual expenditure was obtained for the twelve months and sorted by size. From the total health expenditure, we estimate the out of pocket and the reimbursement to the generic enrollees based on models (48), (50) and (51). For the purpose of this study, we make some simplifying actuarial assumptions because the dataset does not provide information on deductibles and stop loss. $\delta=1000, \mathrm{P}=1 / 4, \sum=5000$ and based on these assumptions, we compute $h=1700$.

Table 1: Estimation of out-of-pockets and reimbursements 
Ogungbenle Gbenga Michael/Mathematical Techniques of Modelling the Out of Pocket with Deductible ...

\begin{tabular}{lllll}
600.00 & 600.00 & 0.00 & 600.00 & 1.00 \\
700.00 & 700.00 & 0.00 & 700.00 & 1.00 \\
800.00 & 800.00 & 0.00 & 800.00 & 1.00 \\
900.00 & 900.00 & 0.00 & 900.00 & 1.00 \\
1200.00 & 1050.00 & 150.00 & 1200.00 & 0.88 \\
2000.00 & 1250.00 & 750.00 & 2000.00 & 0.63 \\
3400.00 & 1600.00 & 1800.00 & 3400.00 & 0.47 \\
4400.00 & 1850.00 & 2550.00 & 4400.00 & 0.42 \\
5300.00 & 2075.00 & 3225.00 & 5300.00 & 0.39 \\
6200.00 & 2300.00 & 3900.00 & 6200.00 & 0.37 \\
7800.00 & 2700.00 & 5100.00 & 7800.00 & 0.35 \\
8400.00 & 2850.00 & 5550.00 & 8400.00 & 0.34 \\
9200.00 & 3050.00 & 6150.00 & 9200.00 & 0.33 \\
10400.00 & 3350.00 & 7050.00 & 10400.00 & 0.32 \\
10600.00 & 3400.00 & 7200.00 & 10600.00 & 0.32 \\
25500.00 & 5000.00 & 20500.00 & 25500.00 & 0.20 \\
34300.00 & 5000.00 & 29300.00 & 34300.00 & 0.15 \\
\hline $\mathbf{1 3 2 2 0 0 . 0 0}$ & $\mathbf{3 8 9 7 5 . 0 0}$ & $\mathbf{9 3 2 2 5 . 0 0}$ & $\mathbf{1 3 2 2 0 0 . 0 0}$ & \\
\hline
\end{tabular}

\section{Discussion of Results}

In table 1, Column 1 is obtained by plugging data into equation (39) and is the same as the sum of columns 2 and 3. This confirms that the aggregate expense is decomposed as $E=z+\pi$ in models (48), (50) and (51). Column 2 is the amount each enrollee has paid out of pocket and consequently the enrollees will be incurring extra bills probably on medical tests and buying medicine in the private pharmacy to further their treatment in public sector health care facilities. This could be required if the public sector health facility does not have such required medicine in the pharmacy or that the public sector facility does not offer such health services. These bills are paid simultaneously as other official payments to government health officials who also probably engage in legally approved private health practice during the official off-duty periods. These payments categorized as out-of-pocket have been estimated using the same data source used in estimating total expenditure. Column 3 represents the amount reimbursed by health insurance scheme and it is the difference between total expenses and out of pocket spending. From table 1, we see that the out of pocket equates to expenses less than deductible but equal to the stop loss where expenses exceed the function value $h$. Furthermore, the stop loss coverage assumption is written to impose restriction on an enrollee's exposure to losses on the underlying scheme. Column 5 is out-of-pocket expressed as a percentage of the health expenditures. From the results in column 5 of same table, the share funded by out of pocket tends to be very high translating to inequality and inadequate coverage in health insurance program. This could result in inordinate spending and eventually place a burden on enrollees with limited economic resources and hence not allowing them to access the needed health care. Therefore, the out-of-pocket becomes a weak instrument in funding health insurance program and its pressure would be felt on low income average enrollees and would be related to a large risk of enrollee's poverty level through extreme costs. The level of health expenditure in column 1 and the distribution of out-of-pocket spending in column 2 change over the enrollees. These changes could be driven by differences in the level of health cover, age, sex, delivery of health care services, income status, severity of health conditions and type of health care provider. From the table 1, the positive relationship between out-of-pocket spending in column 2 and total expenditure in column 1 could occur as a result of adverse selection where enrollees having high health risk conditions or having some unobservable health characteristics are mostly likely to enroll in health insurance. This could introduce serious strain on the out of pocket spending estimations. However, there is inverse relationship between health expenditure and the proportion funded by out-of-pocket. As the health expenditure increases from 500 to 34,300, the share of out- 
of-pocket spending as a percentage of total expenditure progressively reduces from $100 \%$ to $15 \%$. The results show evidence of the necessity to widen health insurance cover for those found with relatively high out-ofpocket spending. Furthermore, interventions should be entrenched in ensuring the sustainability of this scheme as a way to attain affordable health care such as putting policies in place to checkmate the rising out of pocket spending, furnishing financial protection, removal of user fees and overseeing the set policy goals. We should be aware of the danger of double counting that may likely result when health insurance policies reimburse enrollees on medical expenses instead of paying the health care provider directly because enrollees may report expenditures as out of pocket spending. The rising share of public costs on health with growing earning levels describes the high-risk pooling via contributions for compulsory national health insurance program. The share is often associated with the national social-economic growth in tandem with financial and labour markets.

\section{Conclusion}

Accurate estimation of the out-of-pocket spending component of health finance poses a core challenge in different health settings because they represent the biggest source of health finance. Pitacco's approach to out of pocket spending has alternatively improved the current methodologies of estimating out-of-pocket through household survey without any actuarial justification by leveraging on the actuarial practices. As observed in the calculation, the out-of-pocket depends on the expenditure, deductible and stop loss. While enrollees pay contributions regardless of health service use, they assume responsibilities for cost sharing in the form of deductibles only when services are used. Increased cost sharing is being perceived as a technique of transferring costs to those who use the services hence limiting the shared risk and constrain growth in contribution. However, increased cost sharing reduces the value of the cover at the same time contribution rise and consequently enrollees ultimately spend more for less because of exposure to high out-of-pocket cost. The health insurance cost is a function of the kind of cover offered, that is, the level of cover in terms of medical treatment and amounts. The uncertainty in cost represents the contribution which would precisely satisfy the expected cost of the health risk under cover while implicit costs such as commission, management expenses and contingency loading are ignored. The paper used data from public primary healthcare facility, consequently, there is likelihood that the out-of-pocket estimations in private health facilities or higher levels of health care may be different, the influence of actuarial assumptions on out-of- pocket payment could be over-projected in this study. This notwithstanding, this study has provided reliable evidence on the actuarial contribution of health insurance scheme on out-of-pocket spending.

Finally, it is observed that the out of pocket estimating model used did not address issues relating to expected out-of-pocket and expected reimbursements. These gaps were identified and discussed to form part of our results.

\section{References}

[1] Asheim, G.B., Emblem, A.W., \& Nilssen, T. (2004). Deductibles in health insurance: Pay or pain?. International Journal of health care finance and economics, 3: 253-266.

[2] Barber, S.L., Lorenzoni, L., \& Ong, P. (2019). Price setting and price regulation in health care: Lessons for advancing universal health coverage. World health organization, organization for economic cooperation and development. License: CC BY-NC-SA 3.0 IGO.

[3] Bhattacharjya, A.S., \& Sapra, P.K. (2008). Health insurance in China and India: Segmented roles for public and private financing. Health Aff(Millwood), 27(4): 1005-1015.

[4] Duncan, I. (2018). Health care risk adjustment and predictive modeling. ACTEX Learning, 2nd ed., New Hartford p. 3-4.

[5] Faden, L., Vialle-Valentin, C. Ross-Degnan, D., \& Wagner, A. (2011). WHO/HAI project on medicine prices and availability. Review series on pharmaceutical pricing policies and interventions working paper 2: The role of health insurance in the cost-effective use of medicines. 
Ogungbenle Gbenga Michael/ Mathematical Techniques of Modelling the Out of Pocket with Deductible ...

[6] Jack, M., \& Ormiston, M.B. (1999). Analyzing demand for deductible insurance. Journal of risk and Uncertainty. 18: 223-230.

[7] Kaplan, J.T. (2015). Analysing the structure and nature of medical scheme benefit design in South Africa, Phd thesis in actuarial science, University of Cape Town.

https://open.uct.ac.za/bitstream/item/19394/thesis_com_2015_kaplan_josh_tana.pdf?sequence=1, accessed November, 2020.

[8] Kaplan, J., \& Ranchod, S. (2015). An actuarial perspective on medical scheme benefit design presented at the Actuarial Society of South Africa's 2015 Convention 17-18 November 2015, Sandton Convention Centre. https://actuarialsociety.org.za/convention/convention2015/wp-content/uploads/2015/10/2015KaplanRanchod.pdf, accessed November, 2020.

[9] Manning, W., \& Marquis, M. (1996). Health insurance: the trade-off between risk pooling and moral hazard. Journal of health Economics, 15: 609-639.

[10] Martin, S. (2001). The effect of variable health insurance deductibles on demand for physician visits. Health economics, 10(5): 441-456.

[11] Ogungbenle, M.G., Adeyele, J.S., \& Mesioye, A.E. (2020). Modeling moments of insurance claim size under Dirac-delta function. Daffodil International University Journal of Science and Technology, 15(1):43-51.

[12] Onwujekwe, O. E. Uzochukwu B.S., Ezeoke, O.P. \& Uguru, N.P. (2012). Health insurance: principles models and the Nigerian national health insurance scheme. International Journal of medicine and health development, 1118-2601. http://dx.doi.org/10.4314/icm.v16i1.8, accessed November, 2020.

[13] Pakhare, A.P. (2009). A study of determinants influencing development of community based health insurance programme with reference to morbidity patterns, health seeking behavior, and out- ofpocket health expenditure in urban slum inhabitants in a metropolitan city. M.D dissertation in community medicine of the Maharashtra University of health Sciences, Nashik.

[14] Pitacco, E. (2017a). Health insurance: Products and basic actuarial models, University of Zagreb, http://aktuari.math.pmf.unizg.hr/docs/Health_Ins.pdf, accessed November.

[15] Pitacco, E. (2017b). Health insurance: Actuarial aspects, university of Trieste, Italy. https://actuaries.asn.au/Library/Events/Insights/2012/InsightsPresentation- Risk Rated Healthand Sickness Insurance Workshop.pdf

[16] Rao, S. (2004). Health insurance concepts, issues and challenges. Economic and political weekly, August 21:3835-3844.

[17] Ramjee, S., Vieyra, T., Abraham M., Kaplan J., \& Taylor, R. (2014). National health insurance and South Africa's Private Sector, South African Health Review, 1:93-105.

[18] Schlesinger, H. (1985). Choosing a deductible for insurance contracts: Best or worst insurance Policy. Journal of Risk and Insurance, 52(3):522-527.

[19] Thogerson, J. (2016). Optimal premium as a function of deductible: Customer analysis and portfolio characteristics, Risks, 4(42):1-19.

[20] Tse, Y.K. (2009) .Non-life actuarial models, Cambridge University Press, U.K, p. 66-75.

[21] Woodard, J.D., \& Yi, J. (2018). Estimation of insurance deductible demand under endogenous premium rates. Journal of Risk and Insurance, 9999:1-24. 